\title{
Target-Oriented Inversion of Time-Lapse Seismic Waveform Data
}

\author{
Xingguo Huang ${ }^{1, *}$, Morten Jakobsen ${ }^{1,2}$, Kjersti Solberg Eikrem ${ }^{2}$ and \\ Geir Nævdal $^{2}$ \\ ${ }^{1}$ Department of Earth Science, University of Bergen, P.O. Box 7803, 5020 Bergen, \\ Norway. \\ ${ }^{2}$ NORCE Norwegian Research Centre, Postboks 22, 5838 Bergen, Norway.
}

Received 27 May 2018; Accepted (in revised version) 2 March 2019

\begin{abstract}
Full waveform inversion of time-lapse seismic data can be used as a means of estimating the reservoir changes due to the production. Since the repeated computations for the monitor surveys lead to a large computational cost, time-lapse full waveform inversion is still considered to be a challenging task. To address this problem, we present an efficient target-oriented inversion scheme for time-lapse seismic data using an integral equation formulation with Gaussian beam based Green's function approach. The proposed time-lapse approach allows one to perform a local inversion within a small region of interest (e.g. a reservoir under production) for the monitor survey. We have verified that the T-matrix approach is indeed naturally target-oriented, which was mentioned by Jakobsen and Ursin [24] and allows one to reduce the computational cost of time-lapse inversion by focusing the inversion on the target-area only. This method is based on a new version of the distorted Born iterative T-matrix inverse scattering method. The Gaussian beam and T-matrix are used in this approach to perform the wavefield computation for the time-lapse inversion in the baseline model from the survey surface to the target region. We have provided target-oriented inversion results of the synthetic time-lapse waveform data, which shows that the proposed scheme reduces the computational cost significantly.
\end{abstract}

AMS subject classifications: 81U40, 74J25, 74J20, 45Dxx

Key words: Waveform inversion, time-lapse seismic, seismic inverse scattering, target-oriented inversion, Gaussian beam based Green's function.

*Corresponding author. Email addresses: Xingguo.Huang@uib.no, xingguo.huang19@gmail.com (X. Huang), Morten. Jakobsen@uib.no (M. Jakobsen), kjei@norceresearch.no (K. S. Eikrem), gena@norceresearch.no (G. Nævdal) 


\section{Introduction}

Full waveform inversion (FWI) is a powerful tool for reconstructing the subsurface structure and estimating the physical parameters, e.g. P- and S- wave velocities in the subsurface [1]. Advances in full waveform inversion make it possible to do the time-lapse seismic full waveform inversion. Time-lapse seismic is a widely used tool for the dynamic reservoir monitoring and assessing the reservoir changes due to production [2-4]. Recent studies have shown the applicability of the full waveform inversion for the timelapse seismic problem [5-10].

Essentially, the seismic full waveform inversion can be viewed as a seismic inverse scattering problem since the scattering theory provides the relations between the model parameter perturbation and the seismic waveform [1,11-14]. Seismic scattering method is an important technique for seismic data processing, in which the scattered wavefield results from a medium perturbation. The perturbation property of the seismic scattering theory renders it useful not only for seismic forward modeling but also for seismic inversion [15-17]. Since the 1980s, the direct inversion approach based on the linearized wave equation using the seismic scattering method has been widely used [18-23].

Jakobsen and Ursin [24] developed the distorted Born iterative T-matrix method (DBIT) for full waveform inversion based on integral equation methods. The underlying idea of this method is to reduce a nonlinear inverse scattering problem to a sequence of linear inverse scattering problems. For this method, there are several important features: (1) the sensitivity matrix is expressed explicitly in terms of the Green's functions, which is helpful to reduce the computational cost [24,25]; (2) this method can be applied to the cases with multiple sources; (3) the computational cost and convergence problems can be addressed by the T-matrix approach by domain decomposition and renormalization methods $[24,27-29,61]$. These features make the distorted Born iterative T-matrix method more applicable to seismic full waveform inversion. Additional works on this method can be found in Jakobsen and $\mathrm{Wu}[29,31]$ and Wang et al. [32]. Recently, the integral equation formulations were applied to the time-lapse seismic data and to estimate the uncertainty [33]. However, a major limitation of the time-lapse full waveform inversion is that the computational cost is expensive.

The main purpose of this paper is to develop a fast waveform inversion scheme for the time-lapse inversion. We develop a target-oriented inversion method, which is based on the idea of local inversion. Thus, if we develop a fast repeat-inversion scheme, which is only for a small region, the computational cost can be significantly reduced. It makes sense because the effects of the production on the reservoir changes are considered as small perturbations of the earth model [34]. Several studies on the localized full waveform inversion have been proposed to approach this topic. Borisov et al. [35] used the finite-difference injection method to develop an efficient 3-D time-lapse full waveform inversion. Willemsen et al. [36] derive a local solver for full waveform inversion of a small region of interest. Malcolm and Willemsen [37] have developed local solvers for 
localized inversion. Broggini et al. [38] derive the immersive boundary conditions for local wavefields computation. Willemsen and Malcolm [39] applied the coupled acousticelastic local solver to phase inversion. Yuan et al. [10] performed a localized waveform inversion time-lapse survey by combining the wavefield injection and extrapolation. Unlike the methods above, we develop a target-oriented waveform inversion scheme based on the distorted Born iterative T-matrix method [24] and Gaussian beam based Green's function [40-47].

The integral equation formulation of full waveform inversion provides significant advantages over other methods for the target-oriented inversion since it allows the sensitivity matrix to be expressed explicitly in terms of the Green's functions. The distorted Born iterative T-matrix method was introduced as a general FWI method by Jakobsen and Ursin [24]; but they suggested that it could be very suitable for time-lapse inversion, since the T-matrix approach is naturally target-oriented, in the sense that the inversion can be focused on any target if the rest of the model is assumed known. In this paper, we have performed a numerical study of time-lapse inversion based on the distorted Born iterative T-matrix method, to verify that this method reduce computational time for time-lapse inversion. The use of Gaussian beam based Green's functions for the static Reference medium also represent a novel aspect of the present study. The target-oriented inversion method of this paper has the following advantages: (1) When using integral equation methods, it is only necessary to discretize the target area if the Green's functions for the rest of the model is known. This is in contrast to the finite difference method where it is required to discretize the whole model, unless special grid injection method is used; (2) The scattering volume V in the T-matrix formulation is flexible and can be equal to the target area in time-lapse inversion; (3) Another advantage if that compared to finite difference method, there is no grid dispersion error which exists in numerical differential equation solvers, and the integral equation method has a smaller accumulated error [48]. For our approach, two critical aspects are (1) to calculate the initial local wavefields of the target region and (2) its iterative updating for the local inversion. To this end, we employ two methods, called Gaussian beam based Green's function approach [40,45] and the T-matrix method. These two methods are used to calculate the initial local wavefields of the target region. For the updating of the wavefields in the local inversion, we use the T-matrix method. The T-matrix method is from quantum mechanical scattering theory [50-55]. Since its introduction into rock physics, the T-matrix method [29,56-58] has been successfully used to solve the seismic scattering forward problem. More recently, this approach has been extended to seismic inversion [24].

The paper is organized as follows: we first review the seismic scattering forward problem including the Lippmann-Schwinger equation and the T-matrix approach. Then, we review the seismic inverse scattering method for waveform inversion. Further, we present our target-oriented inversion scheme for time-lapse seismic data in Section 4. The new aspects of the paper is described in Section 4 called Time-lapse inversion. Both Sections 4.1 and 4.2 are new compared with the work of [24], but the sequential and double difference strategies are less new than the target-oriented aspects discussed in 
Sections 4.2. Finally, we give the inverted results for the full baseline model and the target-oriented inverted results for the time-lapse data.

\section{Seismic direct scattering problem}

For the inversion of the time-lapse waveform data, we use the seismic scattering method to extrapolate the wavefield. In this section, we review the Lippmann-Schwinger equation, Green's operators and T-matrix theory.

\subsection{The Lippmann-Schwinger equation}

The Green's function for the scalar wave equation in the frequency domain satisfies [59]

$$
\left(\nabla^{2}+\frac{\omega^{2}}{c^{2}(\mathbf{r})}\right) G\left(\mathbf{r}, \mathbf{r}^{\prime}, \omega\right)=-\delta\left(\mathbf{r}-\mathbf{r}^{\prime}\right)
$$

where $c(\mathbf{r})$ is the seismic wave velocity, $\mathbf{r}$ is the position vector, $\mathbf{r}^{\prime}$ is the source position vector, and the Dirac delta function $\delta\left(\mathbf{r}-\mathbf{r}^{\prime}\right)$ represents a unit point source at position $\mathbf{x}^{\prime}$ and $G\left(\mathbf{r}, \mathbf{r}^{\prime}, \omega\right)$ is the Green's function with an angular frequency $\omega$. We can decompose the actual medium into a background medium and a perturbed medium as

$$
\chi(\mathbf{r})=\frac{1}{c^{2}(\mathbf{r})}-\frac{1}{c_{0}^{2}(\mathbf{r})}
$$

where $c_{0}(\mathbf{r})$ is the seismic wave velocity in an arbitrary heterogeneous background medium and $\chi(\mathbf{r})$ is the contrast function. Substituting Eq. (2.2) into Eq. (2.1) yields

$$
\left(\nabla^{2}+\frac{\omega^{2}}{c_{0}^{2}(\mathbf{r})}\right) G\left(\mathbf{r}, \mathbf{r}^{\prime}, \omega\right)=-\delta\left(\mathbf{r}-\mathbf{r}^{\prime}\right)-\omega^{2} \chi(\mathbf{r}) G\left(\mathbf{r}, \mathbf{r}^{\prime}, \omega\right) .
$$

The Lippmann-Schwinger equation can be obtained from Eq. (2.3) as [24,59]

$$
G\left(\mathbf{r}, \mathbf{r}^{\prime}, \omega\right)=G^{(0)}\left(\mathbf{r}, \mathbf{r}^{\prime}, \omega\right)+\omega^{2} \int_{D} d \mathbf{r}^{\prime \prime} G\left(\mathbf{r}, \mathbf{r}^{\prime \prime}, \omega\right) \chi\left(\mathbf{r}^{\prime \prime}\right) G\left(\mathbf{r}^{\prime \prime}, \mathbf{r}^{\prime}, \omega\right)
$$

where $G^{(0)}\left(\mathbf{r}, \mathbf{r}^{\prime}, \omega\right)$ is the Green's function for the background medium, D is the scattering domain where $\chi\left(\mathbf{r}^{\prime \prime}\right)$ is non-zero, and the background Green's function $G^{(0)}\left(\mathbf{r}, \mathbf{r}^{\prime}, \omega\right)$ satisfies the scalar wave equation

$$
\left(\nabla^{2}+\frac{\omega^{2}}{c_{0}^{2}(\mathbf{r})}\right) G^{(0)}\left(\mathbf{r}, \mathbf{r}^{\prime}, \omega\right)=-\delta\left(\mathbf{r}-\mathbf{r}^{\prime}\right) .
$$

If the background medium is homogeneous, the background Green's function $G^{(0)}\left(\mathbf{r}, \mathbf{r}^{\prime}, \omega\right)$ can be calculated by analytic methods. If the background medium is inhomogeneous, the background Green's function can be calculated by ray theory [40] or finite 
difference method [1]. In this work, we employ the Gaussian beam summation method to calculate the background Green's function. Following the recent approach [45], the method implements an estimate of the Green's function in the inhomogeneous background medium that requires (1) a dynamic ray tracing for Gaussian beam computation, (2) the Green's function calculation by Gaussian beam summation. The reason why we use the Gaussian beam is that (1) we can start the inversion with a smoothed inhomogeneous background model; (2) the boundary reflection can be reduced. The details of the method are shown in Appendix A. For the use of the operator form in the following section, we rewrite the Lippmann-Schwinger equation (2.4) in a form of a product of continuous matrices

$$
G\left(\mathbf{r}, \mathbf{r}^{\prime}, \omega\right)=G^{(0)}\left(\mathbf{r}, \mathbf{r}^{\prime}, \omega\right)+\int_{D} d \mathbf{r}_{1} d \mathbf{r}_{2} G^{(0)}\left(\mathbf{r}_{1}, \mathbf{r}_{2}, \omega\right) V\left(\mathbf{r}_{1}, \mathbf{r}_{2}\right) G\left(\mathbf{r}_{2}, \mathbf{r}^{\prime}, \omega\right),
$$

where $V\left(\mathbf{r}_{1}, \mathbf{r}_{2}\right)=\omega^{2} \chi\left(\mathbf{r}_{1}\right) \delta\left(\mathbf{r}_{1}-\mathbf{r}_{2}\right), V$ is a local scattering potential operator, which can be represented by a diagonal matrix in coordinate representation $[29,31]$.

\subsection{The Green's operators and T-matrix approach}

Here, we rewrite the Lippmann-Schwinger equation (2.6) in operator notation

$$
G=G^{(0)}+G^{(0)} V G \text {. }
$$

Following the recent works [24,56-58], we introduce the T-matrix approach into the full waveform inversion approach. Referring to Jakobsen and Ursin [24], we introduce the T-matrix approach by

$$
V G=T G^{(0)},
$$

where represents the T-matrix. Applying the relation (2.8) to the Lippmann-Schwinger equation (2.7), we have

$$
G=G^{(0)}+G^{(0)} T G^{(0)} .
$$

For the Green's operators above, we haven't specified any matrix elements in any particular representation. However, for application to the seismology, we introduce the restricted Green's operators. Note that the above equation is independent of the sourcereceiver configuration. By applying the relation (2.8) to the Lippmann-Schwinger equation (2.7), we have

$$
G_{V S}=G_{V S}^{(0)}+G_{V V}^{(0)} T G_{V S}^{(0)}
$$

where $G_{V S}^{(0)}$ and $G_{V S}$ are the source-dependent Green's functions in the background and actual media, respectively. Similarly, we get Green's functions from the source to the receiver, volume to volume, the scattering point to the receiver, respectively:

$$
\begin{aligned}
& G_{R S}=G_{R S}^{(0)}+G_{R S}^{(0)} T G_{R V}^{(0)}, \\
& G_{V V}=G_{V V}^{(0)}+G_{V V}^{(0)} T G_{V V}^{(0)}, \\
& G_{R V}=G_{R V}^{(0)}+G_{R V}^{(0)} T G_{V V}^{(0)} .
\end{aligned}
$$


Since in Eq. (2.11) $G_{R S}^{(0)}$ is arbitrary, the T-matrix satisfies the Lippmann-Schwinger type equation [24]

$$
T=V+V G_{V V}^{(0)} T
$$

Eq. (2.12) has the exact solution

$$
T=\left(I-V G_{V V}^{(0)}\right)^{-1} V .
$$

The computation of the T-matrix by matrix inversion can be very costly. The domain decomposition was proposed by Jakobsen and $\mathrm{Wu}[29,31]$ to accelerate the T-matrix approach to seismic full-waveform inversion. Also, if the perturbation is small, we can use an approximated form of the T-matrix [24] to update the background Green's function for every iteration.

\section{Seismic inverse scattering problem}

\subsection{The distorted Born iterative T-matrix inversion method}

The distorted Born iterative T-matrix inversion method of [24,81], which is based on a distorted Born approximation $[60,61]$ and solves for the scattering potential $\mathbf{V}$, uses an iterative scheme that for each iteration updates the scattering potential. Because the T-matrix is used for the Green's function update, the method has been referred to distorted Born iterative T-matrix inversion method. The relationship between a variation $\delta \mathbf{V}^{(i)}=\mathbf{V}-\mathbf{V}^{i}$ in the scattering potential $\mathbf{V}$ around a heterogeneous background model with scattering potential $\mathbf{V}^{(i)}$ and a variation $\delta \mathbf{G}_{R S}=\mathbf{G}_{R S}-\mathbf{G}_{R S}^{(i)}$ in the Green's function $\mathbf{G}_{R S}$ around the Green's function $\mathbf{G}_{R S}^{(i)}$ for the background medium is given by $[60,61]$

$$
\delta \mathbf{G}_{R S}^{(i)}=\mathbf{G}_{R V}^{(i)} \delta \mathbf{V}^{(i)} \mathbf{G}_{V S}^{(i)}
$$

where

$$
\begin{aligned}
& \mathbf{G}_{R S}^{(i)}=\mathbf{G}_{R S}^{(0)}+\mathbf{G}_{R V} \mathbf{T}^{(i)} \mathbf{G}_{V S^{\prime}}^{(0)}, \\
& \mathbf{G}_{V S}^{(i)}=\mathbf{G}_{V S}^{(0)}+\mathbf{G}_{V V} \mathbf{T}^{(i)} \mathbf{G}_{V S^{\prime}}^{(0)} \\
& \mathbf{G}_{R V}^{(i)}=\mathbf{G}_{R V}^{(0)}+\mathbf{G}_{R V} \mathbf{T}^{(i)} \mathbf{G}_{V V}^{(0)}
\end{aligned}
$$

are matrices of Green's functions for the background medium responsible for different parts of the scattering path from the source to the receiver via the volume, and

$$
\mathbf{T}^{(i)}=\mathcal{T}\left(\mathbf{V}^{(i)}\right)
$$

is the corresponding T-matrix. In Eqs. (3.2)-(3.4), the reference Green's functions $\mathrm{G}_{R S}^{(0)}$, $\mathrm{G}_{V S^{\prime}}^{(0)}$, and $\mathrm{G}_{R V}^{(0)}$ are calculated for a heterogeneous medium with Gaussian beams and the 
background Green's functions $\mathbf{G}_{R S}^{(i)}, \mathbf{G}_{V S}^{(i)}$, and $\mathbf{G}_{R V}^{(i)}$ (typically calculated numerically for an inverted model with scattering potential $\mathbf{V}^{(i)}$ relative to the reference model with zero scattering potential) are static and dynamic, respectively. By dynamic, they are updated after each linearised inversion step.

The observable scattered (data residual) wavefield $\delta d_{R}^{(i)}$ associated with the perturbation $\mathbf{V}^{(i)}$ can be written as

$$
\delta d_{R}^{(i)}=\delta \mathbf{G}_{R S}^{(i)} \mathbf{s},
$$

where $\mathbf{s}$ is an $N_{\mathcal{S}}$-dimensional vector associated with the source functions at $N_{S}$ different source positions. By combining Eqs. (3.1) and (3.6), a linear relation between the scattered (data residual) field $\delta d_{R}^{(i)}$ and the scattering potential variation (or perturbation) $\mathbf{V}^{(i)}$ [24] can be obtained:

$$
\delta d_{R}^{(i)}=\mathbf{G}_{R V}^{(i)} \delta \mathbf{V}^{(i)} \mathbf{G}_{V S}^{(i)} \mathbf{s}
$$

From Eq. (3.7), one can observe that when the scattered (data residual) field $\delta d_{R}^{(i)}$ is known, one can determine the perturbation $\mathbf{V}^{(i)}$ using a regularised least-squares inversion method. Then, the original $\mathbf{V}^{(i)}$ can be replaced with the inverted scattering potential and more accurate solutions can be obtained with iterations in a direct iterative manner. It should be noted that the data residual field and background medium Green's functions can be updated after each iteration by using the exact relations (3.1)-(3.4).

\subsection{Some details for implementation}

In this work, we use the distorted Born iterative method to solve the inverse problem for the baseline and time-lapse inversions. The underlying idea of the distorted Born iterative inversion method is to reduce a nonlinear inverse scattering problem to a series of linear inverse scattering problem.

Eq. (3.7) can also be expressed as [24]

$$
\delta d_{r, s}^{(i)}=\sum_{n=1}^{N} J_{r n, s}^{(i)} \delta \chi_{n}
$$

where the scattered wavefields $\delta d$ represents the difference between the calculated wavefields in the background medium and the wavefields in the actual medium, $\delta \chi$ is the difference of the contrast potential, and the sensitivity matrix can be written as [24]

$$
J_{r n, s}^{(i)}=\left[G_{r n}^{(i)} \delta v_{n} G_{n s}^{(i)}\right] f_{s}
$$

where $G_{r n}^{(i)}$ and $G_{n s}^{(i)}$ are the Green's functions in a dynamic heterogeneous reference medium associated with the receivers and the sources at $i$ th iteration, respectively. The Green's functions that is updated after each iteration and where we compute the corresponding Green's functions by solving the Lippmann-Schwinger equations (3.2)-(3.4). 
Here, $f_{s}$ is the source function associated with the angular frequency, and $\delta v_{n}$ is the Kronecker-delta.

From Eq. (3.8), one can observe that the nonlinear inverse scattering problem has been reduced to a series of linear inverse scattering problem. However, solving inverse problem is an ill-posed problem. In order to obtain a meaningful solution, we can apply generalized Tikhonov regularization to the inverse scattering problem. Thus, the solution can be obtained by solving the following optimization problem [62]

$$
E(\delta \chi)=\left\|\delta d^{(i)}-J^{(i)} \delta \chi\right\|^{2}+\alpha\|\delta \chi\|^{2},
$$

where \|\| represents the $L 2$ norm, $\alpha$ is the regularization parameter, which can be chosen by the cooling scheme [24], a modified version of the method described in Lavarello and Oelze [63] and in Hesford and Chew [64], or L-curve method [65]. The regularization parameter is very important for the inversion. Through many numerical tests, we choose to use the cooling scheme as

$$
\alpha^{(i)}=\alpha_{0} a^{(i-1)},
$$

where $\alpha_{0}$ is the initial value, and $0.1<a<0.9$. However, to obtain a relatively good result, we should choose different initial values in Eq. (3.11) for the baseline and timelapse inversion. The details will follow in Section 4. The iterative form solution for the inversion is

$$
\chi^{i+1}=\chi^{(i)}+\left(H^{i}+\alpha^{(i)} I\right)^{-1} V^{(i)},
$$

with the gradient

$$
V^{(i)}=\Re\left[\left(J^{(i)}\right)^{\dagger} \delta d^{(i)}\right],
$$

where $\delta d^{(i)}$ is the difference between the observed data and the calculated data, + denotes transpose conjugate, $i$ is the number of iteration, $\Re$ represents the real part, and

$$
H^{(i)}=\Re\left[\left(J^{(i)}\right)^{\dagger} J^{(i)}\right]
$$

is the approximate Hessian matrix. The process of solving the nonlinear inverse scattering problem is essentially similar to the Gauss-Newton optimization methods for FWI (e.g. the process for inverse Hessian, shown as Eq. (3.12) in this paper and Eq. (13) in [76]). Similar to the GN Hessian (equation 10 in [76]), the element of the Hessian in our approach is formed by correlating the two Frechet derivative wavefields at the receivers, which is a approximate Hessian. Actually, it has been demonstrated that the Distorted Born iterative method is consistent with the Gauss-Newton methods of optimization (see Remis and van den Berg [74], Oristaglio and Blok [73], Jakobsen and Ursin [24]. However, different from [75] and [76], we construct the approximate Hessian matrix explicitly in terms of Green's functions based on the integral equations. The main difference between our scattering approach and the conventional adjoint state method is that we have 
an explicit representation of the sensitivity matrix in terms of Green's functions, that can easily be updated after each iteration using the variational T-matrix approach, without having to perform a full forward simulation. And also instead of using the conjugate gradient method, we solve a linear system (using Gaussian elimination method) involving our approximate Hessian matrix. Appendix B shows the pseudo code of the algorithm, which is based on the pseudo code of the DBIT inversion algorithm shown in [81].

The above formulations are used to obtain both the full baseline model and the timelapse model. For the baseline inversion, we formulate the Gaussian beam based distorted Born iterative T-matrix inversion method by using the Gaussian beam based Green's function as the background Green's function. This method incorporates several important features: (1) The Gaussian beam has flexibility in calculating the wavefields and the boundary reflection can be avoided because it is a ray-based method. (2) Due to the use of the complex traveltime [40-46] in Gaussian beam, this method can deal with the problem of caustic, which is a limitation of the conventional ray theory. For this inversion, we use the integral equations as wavefield propagators, which is based on scattering theory. The Gaussian beam is only used to compute the background Green's function, but the scattering theory can address all the wavefields, e.g. multiple scattering. In fact, for relatively simple medium, combining the distorted Born iterative inversion method and the Gaussian beam can be used for the baseline inversion. However, this paper focus on the target-oriented time-lapse inversion. To obtain an accurate baseline model, the T-matrix is used in the baseline inversion.

\section{Time-lapse inversion}

\subsection{Sequential and double difference strategies}

After obtaining the baseline model, we can perform the time-lapse inversion using the time-lapse inversion strategies. In this work, we employ two strategies, called sequential difference strategy and double difference strategy.

Sequential difference strategy: Fig. 1 shows the schematic diagrams of the sequential difference method. The sequential difference strategy $[6,7,33]$ considers the baseline model as the initial model. Because the perturbation resulting from the reservoir changes is localized and only occur in a small region, starting from the baseline model for the time-lapse inversion is a good candidate and can reduce the computation cost. After completing the time-lapse inversion, the perturbation can be obtained by a subtraction between the inverted baseline and monitor model.

Double difference strategy: Fig. 2 shows the schematic diagrams of the double difference method. The double difference strategy was proposed by Waldhauser and Ellsworth [66] in the traveltime tomography for improving the earthquake source location $[7,67,68]$. It also starts the time-lapse inversion with the baseline model $[5,7]$. However, instead of a full-data inversion in the sequential difference strategy, the double 


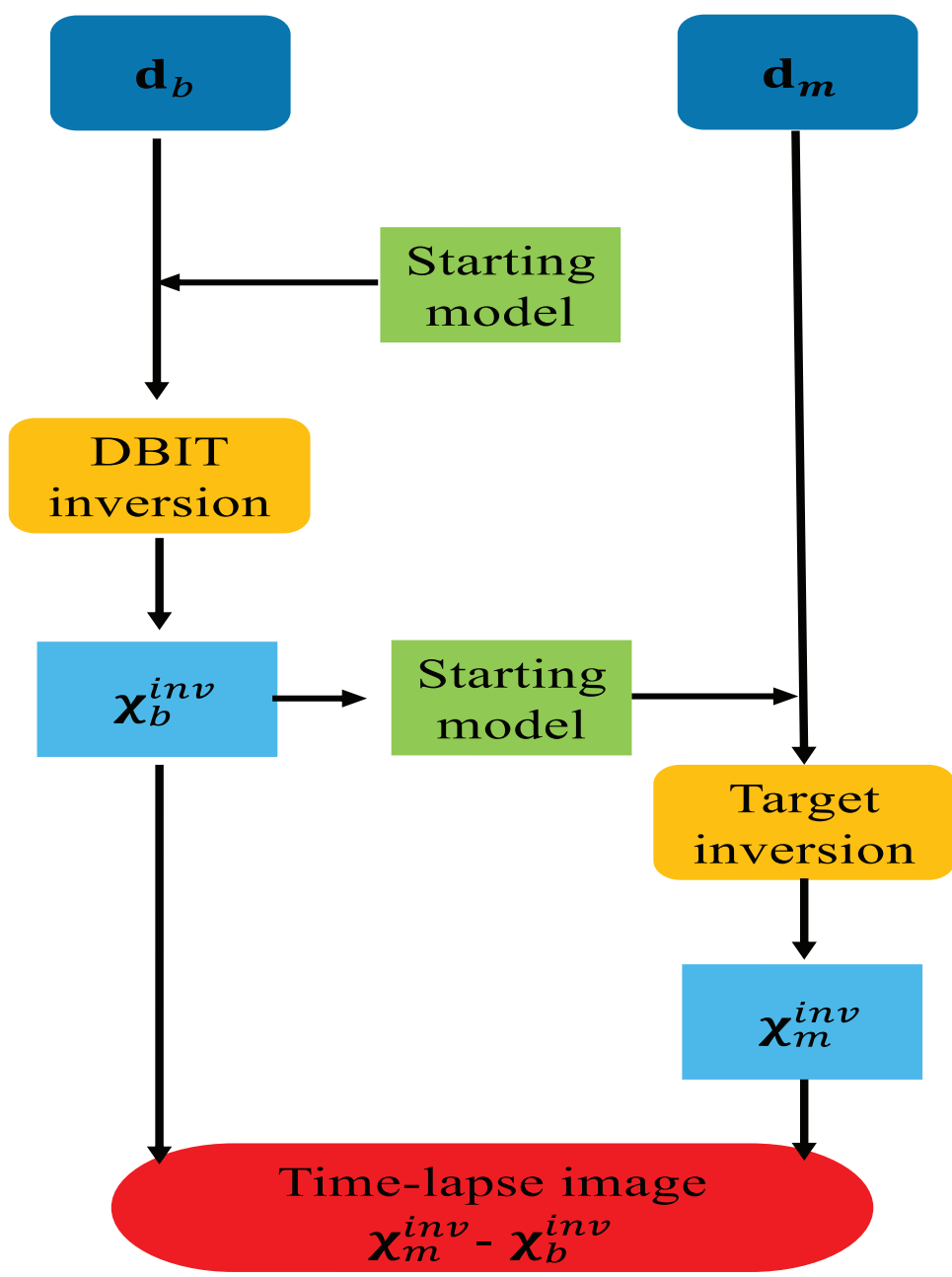

Figure 1: Schematic diagrams of the sequential difference method.

difference strategy only inverts the time-lapse perturbation model using the time-lapse data difference as

$$
E(\delta \chi)=\frac{1}{2}\left\|\left(u_{\text {monitor }}-u_{\text {baseline }}\right)-\left(d_{\text {monitor }}-d_{\text {baseline }}\right)\right\|^{2},
$$

where $u_{\text {monitor }}, u_{\text {baseline, }}, d_{\text {monitor }}$ and $d_{\text {baseline }}$ are the calculated monitor data set, calculated baseline data set, observed monitor data set and observed baseline data set, respectively. The time-lapse data difference consists of two parts: One is the difference between the observed monitor and baseline data set, and the other is the difference between the calculated monitor and baseline data set. Moreover, there is an assumption that the shot and receiver positions are equal in the data sets. 


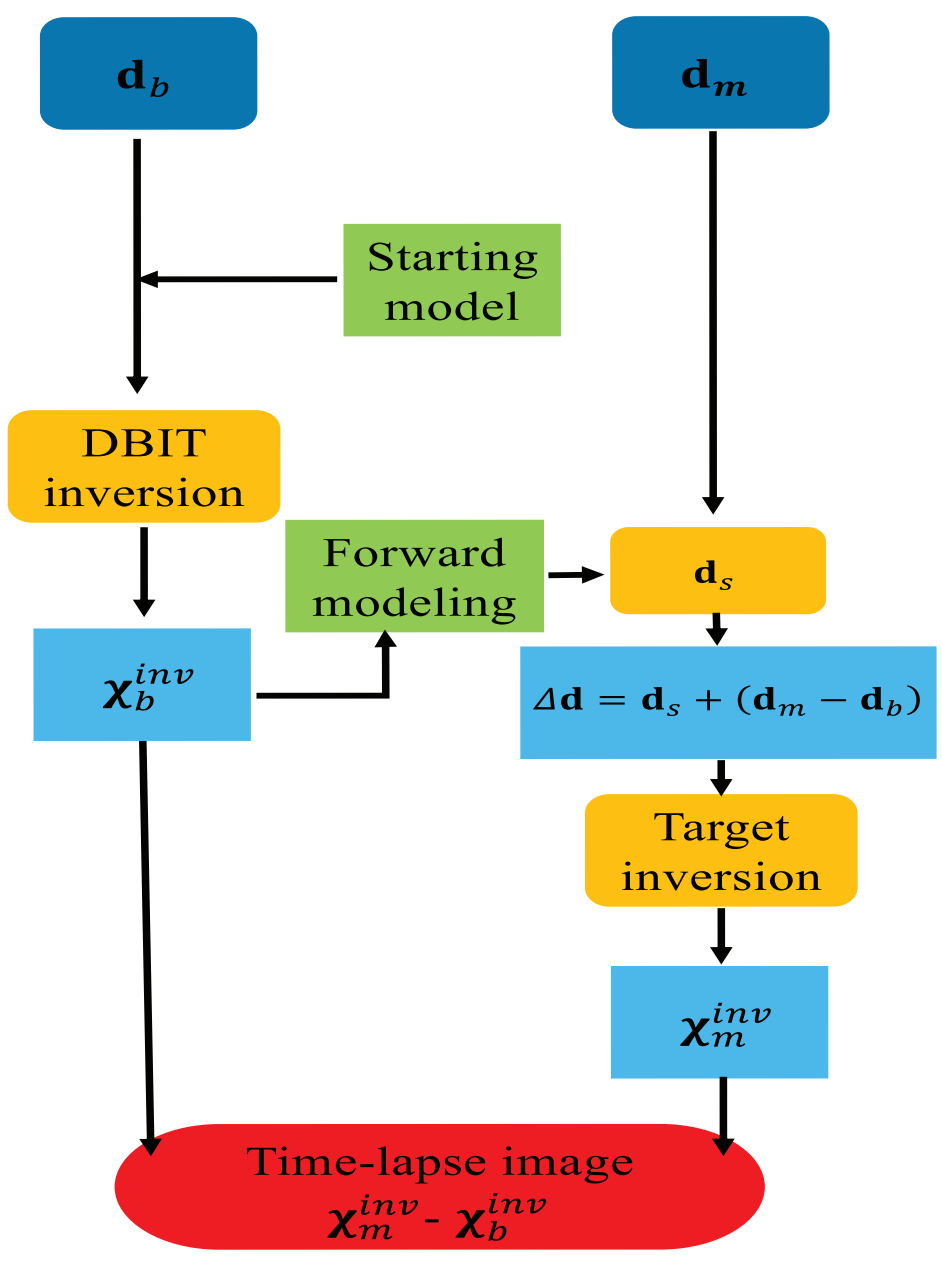

Figure 2: Schematic diagrams of the double difference method.

\subsection{The target-oriented scheme}

To reduce the computational cost of the time-lapse inversion, we develop a targetoriented inversion scheme. The target-oriented concept was proposed to reduce the computational cost of the wave-equation least-squares migration $[69,70]$ and the reverse time migration [71]. This concept is generalized to the local full waveform inversion $[10,35,36]$. The scheme of the local inversion is referred to as the target-oriented inversion.

For the target-oriented inversion, there have been several different approaches to perform localized inversion. Borisov et al [35] used the finite-difference injection method to develop a localized full waveform inversion for time-lapse imaging. In this approach, the wavefields are recorded around the local region, and the initial wavefield of the target region are injected. In subsequent target-oriented inversion, the local wavefields are updated. However, the method suffers a limitation that it does not accurately model the 


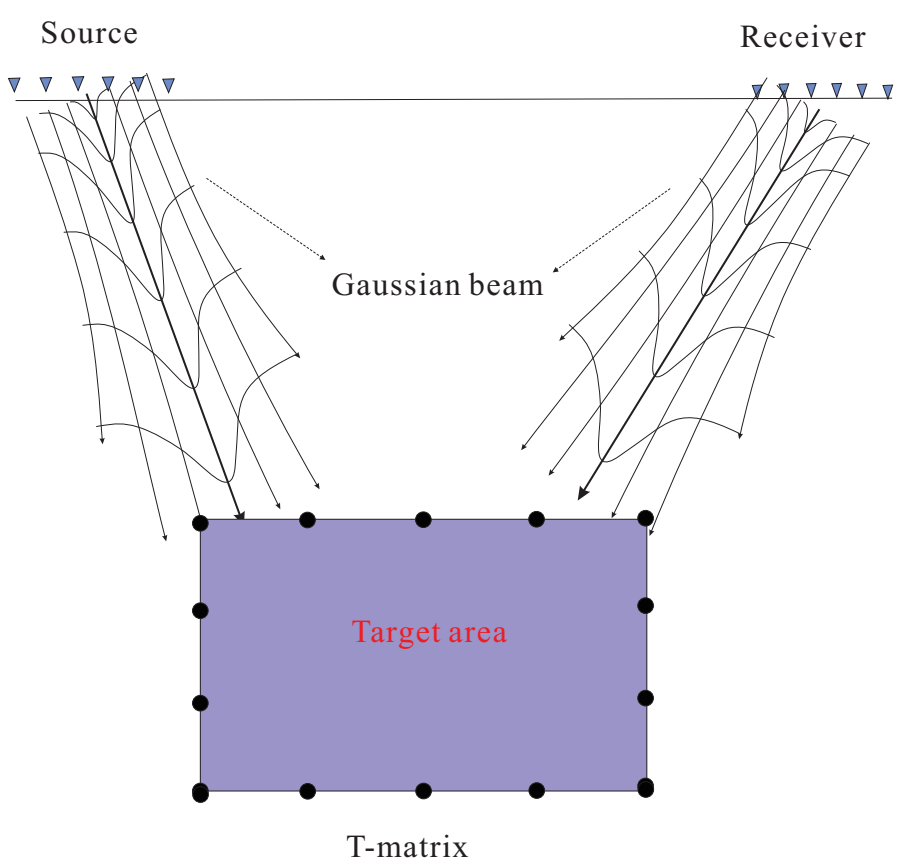

Figure 3: Sketch illustrating the main ideas of the target-oriented inversion. Note that in this scheme, the Gaussian beam and T-matrix are used to propagate the wavefields from the source and receiver and- the wavefields are propagated from the receiver to scattering region by the reciprocity theorem.

higher order long-range interactions between the scattered wavefield propagating into the unaltered exterior domain and then re-enter into the local target domain. Yang et al. [77] developed time-lapse waveform inversion by transforming the original survey into a new survey at the top of the reservoir. The new data sets are synthesized from the recorded data with the re-datumed signals and the new virtual survey geometry. Because of modifying the surface data recordings, they introduce varying degrees of artefacts. Valenciano et al. [70] proposed to explicitly compute an approximation of the Hessian in a target-oriented fashion. After computing a nondiagonal Hessian matrix, they obtain the inverse image using an iterative algorithm. However, this method used the Green's function by solving the one-way wave equation, which has a limited accuracy for large-angle propagation.

Our approach uses integral equations to develop the target-oriented inversion. The method requires only one simulation for the initial Green's functions on the entire subsurface model and the initial Green's functions are computed efficiently since we employ Gaussian beams. For the subsequent target-oriented inversion, the multiple scattering occurring within the target area can be modeled by the T-matrix. The T-matrix approach is naturally target-oriented since the T-matrix refers to the target-region only and is independent of the source-receiver configuration. Our target-oriented inversion is based on the full waveform inversion using the integral equation formulations. Fig. 3 shows the sketch illustrating the main ideas of target-oriented inversion. The underlying idea of 
the proposed target-oriented inversion is that we only need to compute the local wavefields of the target region. The initial local scattered wavefields of the target region are computed in the inverted baseline model.

We have developed our time-lapse inversion method adopting the distort Born iterative T-matrix method described in Section 2.3 and using the inversion formulations described in Section 3. Our inversion approach involves a static heterogeneous reference medium (denoted by $\mathbf{G}_{R S}^{(0)}, \mathbf{G}_{V S}^{(0)}$, and $\mathbf{G}_{R V}^{(0)}$ ) for which we use Gaussian beam based Green's functions, and a dynamic heterogeneous reference medium that is updated after each iteration and where we compute the corresponding Green's functions $\left(\mathbf{G}_{R S}^{(i)}, \mathbf{G}_{V S}^{(i)}\right.$, and $\left.\mathbf{G}_{R V}^{(i)}\right)$ by solving the Lippmann-Schwinger equation. The Green's functions have scattering information from the target area. That means that we could estimate the velocity within the target.

For the time-lapse target inversion scheme, there are three main steps:

(1) Compute the background Green's function in the smoothed baseline model;

(2) Compute the static Green's functions in the inverted baseline model using the Gaussian beam based Green's function and T-matrix from the survey surface to the target region;

(3) Perform the local inversion, the local wavefields are updated by the LippmannSchwinger equations associated with T-matrix approach within the target region.

\section{Numerical results}

\subsection{Baseline inversion}

In this section, we use a subset of the Marmousi $2 \mathrm{P}$ wave velocity model [72] to test the baseline inversion scheme. The model size is $2460 \mathrm{~m} \times 1620 \mathrm{~m}$. The size of the grid is $20 \mathrm{~m} \times 20 \mathrm{~m}$. Fig. 4(a) shows the resampled baseline model. We employ 81 sources and 123 receivers, which are both located at the surface and distributed uniformly from $0 \mathrm{~m}$ to $2460 \mathrm{~m}$. We employ a Ricker wavelet with the central frequency of $7.5 \mathrm{~Hz}$. In this example, we use the sequential frequency inversion scheme, which inverts frequency by frequency. Here, we choose a frequency group of $2 \mathrm{~Hz}, 5 \mathrm{~Hz}, 8 \mathrm{~Hz}, 11 \mathrm{~Hz}, 15 \mathrm{~Hz}$ and $18 \mathrm{~Hz}$. For each frequency, the maximum number of iterations is 30 . We generated the frequency component synthetic data and used the fast Fourier transform with a sampling interval of $0.004 \mathrm{~s}$ and the total record length of $3 \mathrm{~s}$. A key point of this inversion is to choose the regularization parameter. We use the cooling scheme [24] for this test. In the numerical tests, we find that if the regularization parameter is too small in the beginning of the iterative process, artefacts can build-up in the inverted results, to compensate for model errors. This is because in the scattering domain, the initial information about the scattered wavefield is not sufficient. After many tests, we decide to use 10 as the initial value $\alpha_{0}$ of the cooling scheme (see Eq. (3.11)). We choose $a$ in the Eq. (3.11) as 0.9. We 

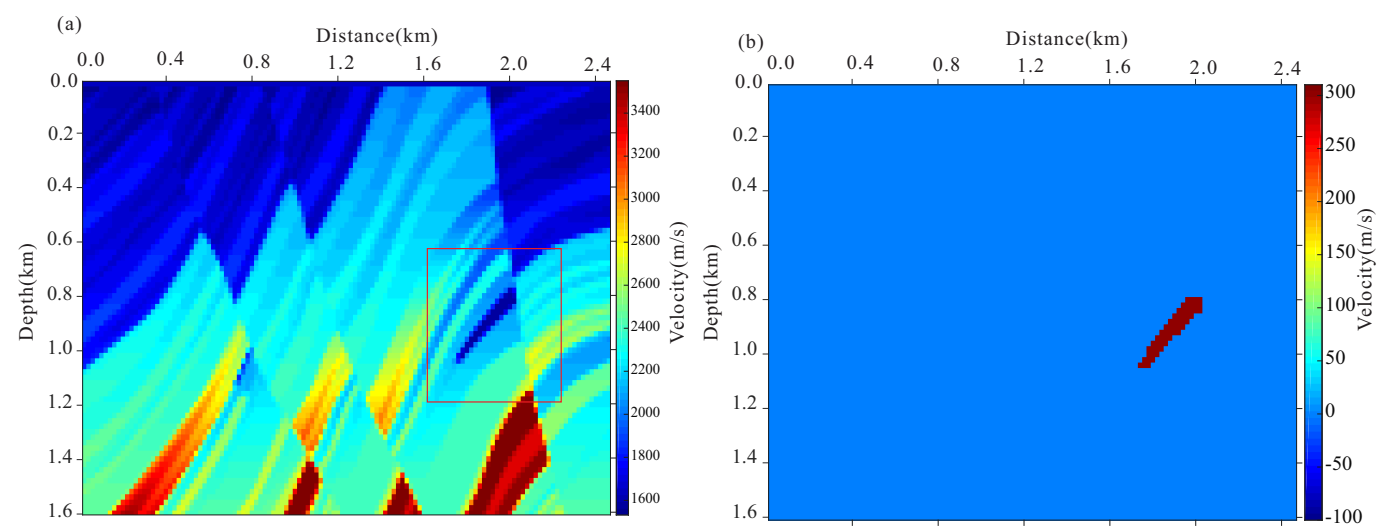

Figure 4: The baseline model and target region. (a) A subset version of the Marmousi2 P- wave velocity model; (b) time-lapse perturbation of $300 \mathrm{~m} / \mathrm{s}$.

have tested with noise-free and noisy data. For the noisy data, the random noise has been added to each frequency component data [24]

$$
\mathbf{d}^{\text {noisy }}=\left(\mathbf{d}+\frac{|\mathbf{d}|}{\sqrt{S N R}} \frac{\eta}{|\eta|}\right)
$$

where $\eta$ is a vector with independent random numbers taken from a Gaussian distribution, and the decibel $(\mathbf{d B})$ is used to measure noise level. We use the signal-to-noise ratio (denoted by SNR) of $30 \mathrm{~dB}$ ( $3 \%$ noise) and $26 \mathrm{~dB}$ (5.1\% noise), respectively.

Fig. 5(a) shows the smoothed starting model for the baseline inversion. For field case, the smoothed starting model can be obtained by traveltime inversion. The Gaussian beams have been computed for this model for the initial Green's function. Figs. 5(b), (c) and (d) show the inverted results for the subset of the Marmousi2 baseline model with a smoothed starting model (shown in Fig. 5(a)) with noise-free data and noisy data with the SNR of $30 \mathrm{~dB}$ and $26 \mathrm{~dB}$, respectively. From Fig. 5(b), one can observe that the model structure can be distinguished clearly. This implies that the inversion method works well for noise-free data. To quantify the errors between the true model and the inversion results, we compute the differences between the true baseline model and the inverted baseline results with noisy data, shown as Fig. 6. From Fig. 6, one can observe that due to the effect of a stronger noise, the error of the inverted results with signal-to-noise ratio of $26 \mathrm{~dB}$ is larger than that with the signal-to-noise ratio of $30 \mathrm{~dB}$.

\subsection{Time-lapse inversion}

In this section, we focus on the target-oriented inversion of the time-lapse model. Fig. 4(b) shows the time-lapse perturbation model. This model is obtained by adding a perturbation of $300 \mathrm{~m} / \mathrm{s}$ to the baseline model. For the numerical tests, we use the same sources and receivers as the baseline inversion. Here, we use the same Ricker wavelet, grid size 

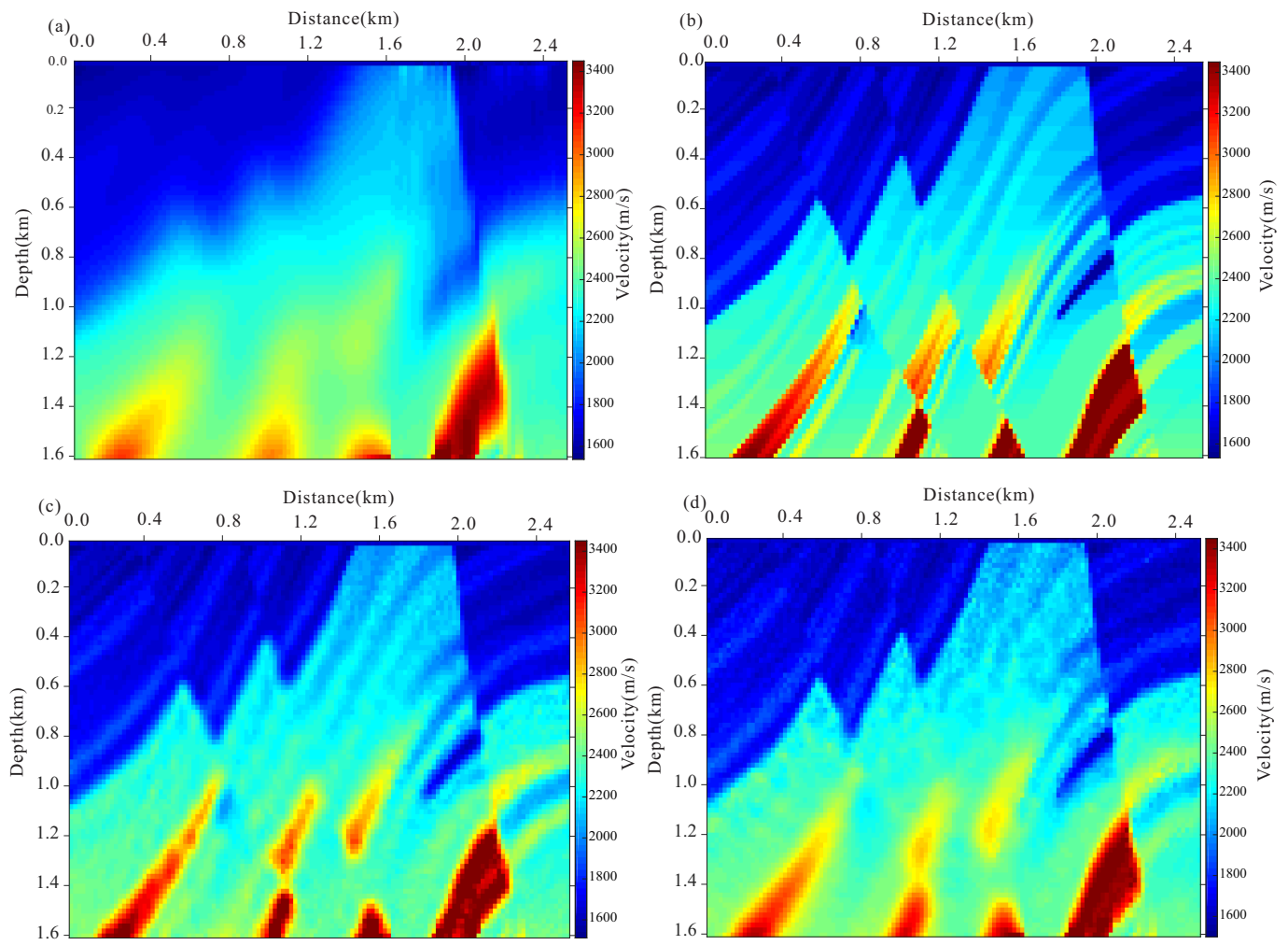

Figure 5: The baseline inversion results using Gaussian beam plus T-matrix method. (a) The smoothed starting model; (b) the inverted result of noise-free data; (c) the inverted result of noisy data (30dB); (d) the inverted result of noisy data $(26 \mathrm{~dB})$.

and frequency group as the baseline inversion. The size of the target region is $600 \mathrm{~m} \times$ $600 \mathrm{~m}$, shown as Fig. 4(b). The locations are from $1600 \mathrm{~m}$ to $2200 \mathrm{~m}$ and from $600 \mathrm{~m}$ to $1200 \mathrm{~m}$ in the horizontal and vertical directions, respectively. For the regularization parameter, we use a modified version of the cooling scheme (Jakobsen and Ursin, 2015). In our approach, the initial value is chosen as $\alpha_{0}=\sqrt{\operatorname{trace}(H) / \text { length }(H)}$, where $H$ represents the Hessian matrix as before. We choose a in the Eq. (3.11) as 0.9. We generated the frequency component synthetic data and used the fast Fourier transform with a sampling interval of $0.004 \mathrm{~s}$ and the total record length of $3 \mathrm{~s}$. The maximum number of iterations is 30 for each frequency.

\subsubsection{Comparison of time-lapse inversion strategies}

To validate the accuracy of our target-oriented inversion approach, we have carried out the numerical experiments on a time-lapse model in which there is a perturbation due the time-lapse effects, shown as Fig. 4(b). For this experiment, we have tested both the sequential difference strategy and double difference strategy. We employ the baseline model used in the last section, which is shown in Fig. 4(a), as the true baseline model. 

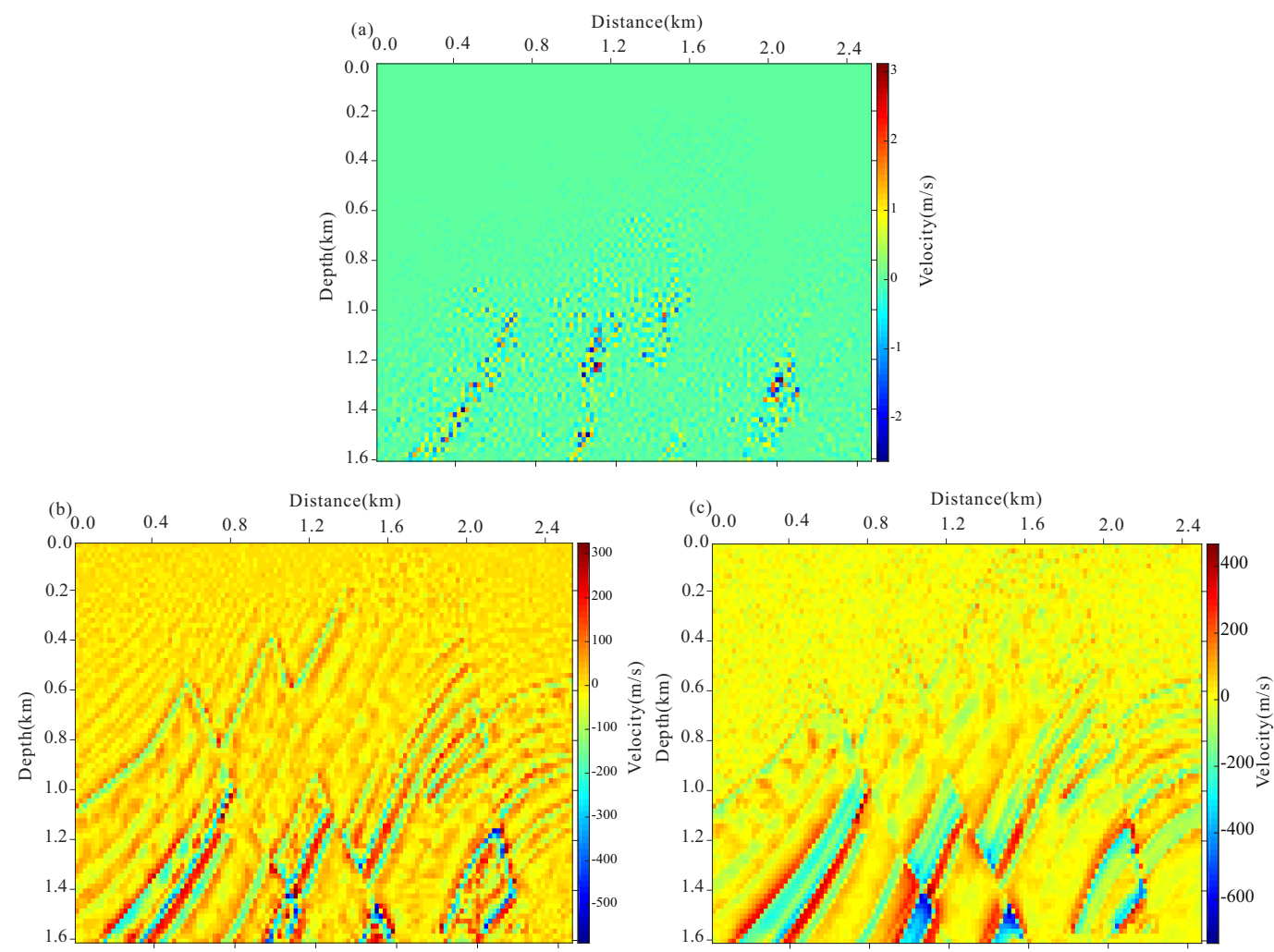

Figure 6: The errors of the baseline inversion. (a) The inversion error with noise-free data; (b) The inversion error with noisy data $(30 \mathrm{~dB})$; (c) the inversion error with noisy data $(26 \mathrm{~dB})$.

We have performed the full-model inversion and target-oriented inversion on this timelapse model. Fig. 7 shows the inverted results of the noise-free data for the time-lapse inversion with the sequential difference strategy and double difference strategy, respectively. All computations were performed on Intel (R) Xeon (R) CPU E5-4650 2.7 GHz. The computational times of the full-model inversion with the sequential difference and double difference strategies are $2.15 \times 10^{4} \mathrm{~s}$ and $2.11 \times 10^{4} \mathrm{~s}$, respectively. The computational times of the target-oriented inversion with the sequential difference and double difference strategies are $418 \mathrm{~s}$ and $431 \mathrm{~s}$, respectively. One can see that by using the target-oriented inversion scheme, the computational cost has been reduced by approximately $80 \%$. Comparison of the inversion results shows that, for both of the sequential and double difference strategies, the results of the target-oriented inversion are slightly better than that of full-model inversion. This can easily be explained. The larger area we have in the inversion, the more errors of the inversion will occur. We can observe from Figs. 8(a) and (c) that for the full-model inversion there are some inversion artefacts outside the target region. This is because the full waveform inversion has the problems of high ill-posedness and nonlinearity. However, the potential artifacts can be avoided in the target-oriented inversion because there is no model update outside the target region. 

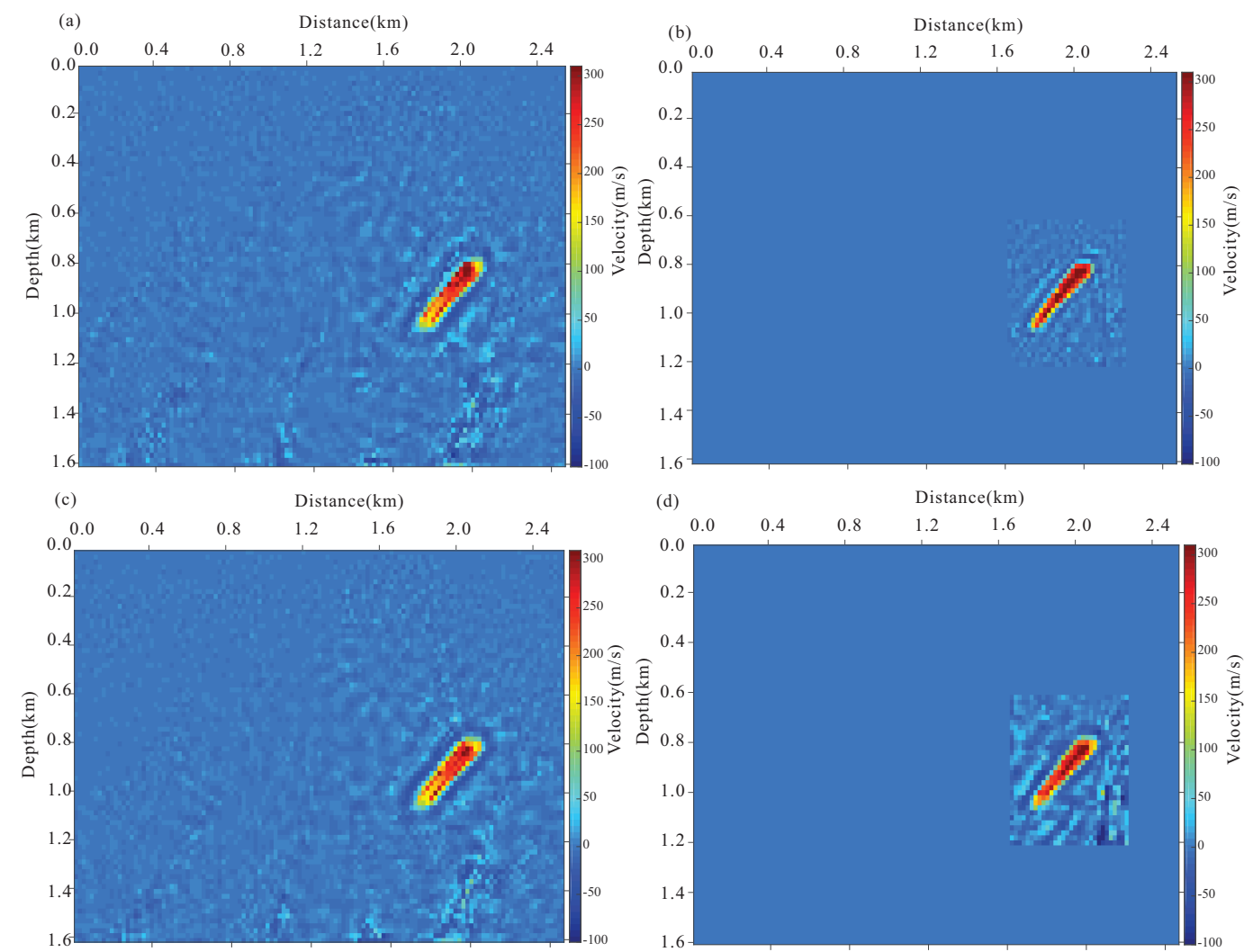

Figure 7: The time-lapse inversion results of the noise-free for a perturbation of $300 \mathrm{~m} / \mathrm{s}$ with the true baseline model as the starting model. (a) The full-model inversion with the sequential difference strategy; (b) the target-oriented inversion with the sequential difference strategy; (c) the full-model inversion with the double difference strategy; (d) the target-oriented inversion with the double difference strategy.

\subsubsection{Inversion of noise-free data with the inverted baseline model}

In this section, we employ the inverted baseline model of the noise-free data (shown as Fig. 4(b)) as the starting model for the time-lapse inversion and use noise-free data for time-lapse inversion. In this inversion, we use both the sequential difference strategy and double difference strategy as well. Fig. 8 shows the inverted results for the timelapse inversion using the full-model scheme (Figs. 8(a) and (c)) and target-oriented inversion scheme (Figs. 8(b) and (d)). We also compare the results of the target-oriented inversion and that of the full-model inversion. From Fig. 8, one can make the following observations: (1) whether for the target-oriented inversion or full-model inversion, the results from the double difference strategy are better than that from the sequential difference strategy, which shows that compared with the sequential difference strategy, the double difference strategy works better; (2) for both the sequential difference and double difference strategy, the target-oriented scheme works nearly the same as the full-model scheme. However, the computational cost can be reduced by target-oriented inversion. 

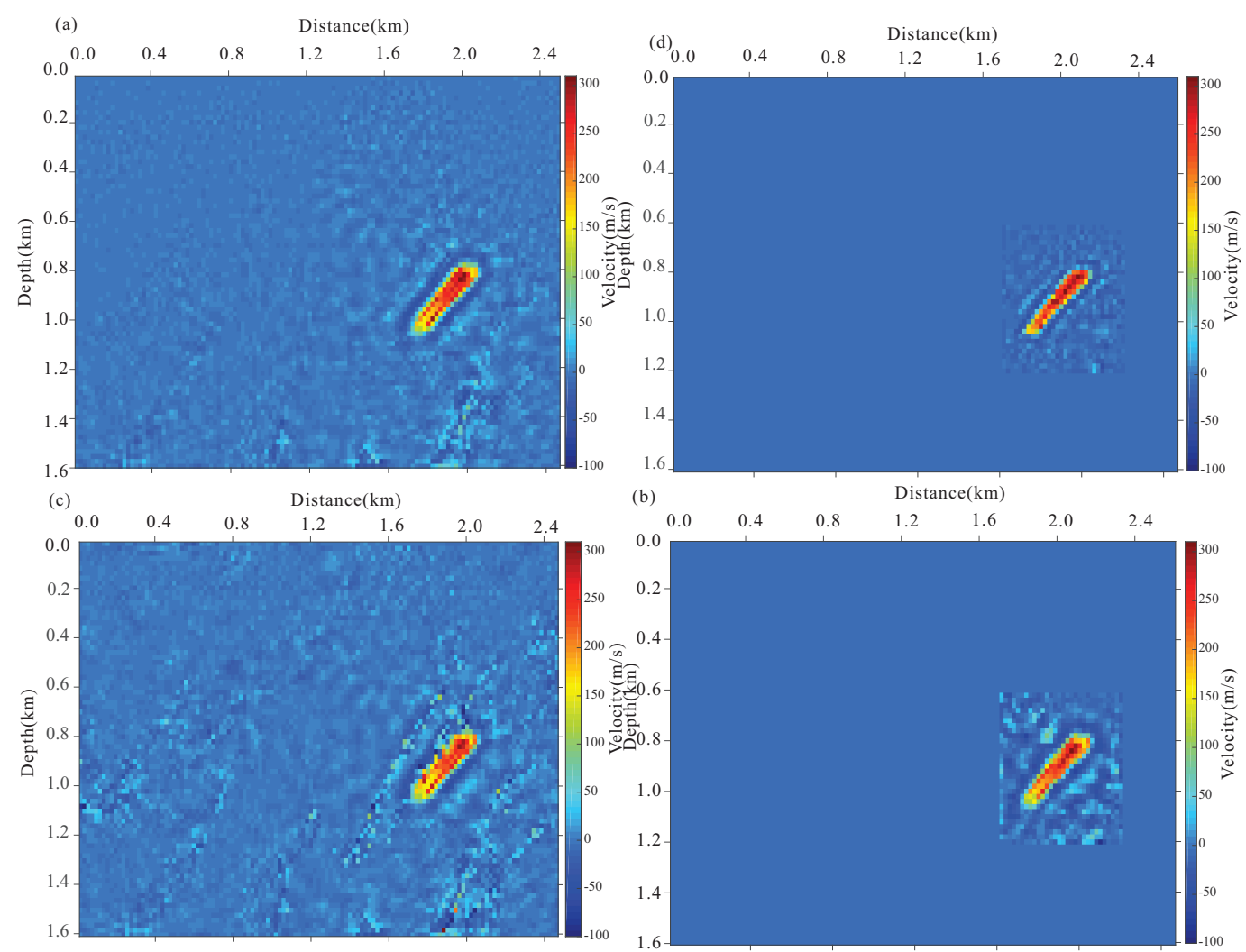

Figure 8: The time-lapse inversion results of noise-free data for a perturbation of $300 \mathrm{~m} / \mathrm{s}$ with the inverted baseline model of noise-free data as the starting model. (a) The full-model inversion with the sequential difference strategy; (b) the target-oriented inversion with the sequential difference strategy; (c) the full-model inversion with the double difference strategy; (d) the target-oriented inversion with the double difference strategy.

The double difference strategy, which inverts with the data difference rather than fulldata, requires the starting baseline model that can accurately describe the kinematics and structures, otherwise the time-lapse energy cannot be localized [7].

\subsubsection{Inversion of noisy data with the inverted baseline model}

To make the numerical experiments more realistic, we perform the numerical tests with the noisy data. Fig. 9 shows the inverted results for the time-lapse inversion of the noisy data $(30 \mathrm{~dB})$ using the full-model scheme (Figs. 9(a) and (c)) and target-oriented scheme (Figs. 9 (b) and (d)). Fig. 10 shows the inverted results for the time-lapse inversion of the noisy data (26 dB) using the full-model scheme (Figs. 10(a) and (c)) and target-oriented scheme (Figs. 10(b) and (d)). For the inversion of the noisy data (26 dB), the computational times of the target-oriented inversion with the sequential difference and double difference strategies are $2.01 \times 10^{4} \mathrm{~s}$ and $2.19 \times 10^{4} \mathrm{~s}$, respectively. The computational times of the target-oriented inversion with the sequential difference and double difference strategies are $246 \mathrm{~s}$ and $602 \mathrm{~s}$, respectively. The computational cost has been reduced from 70 

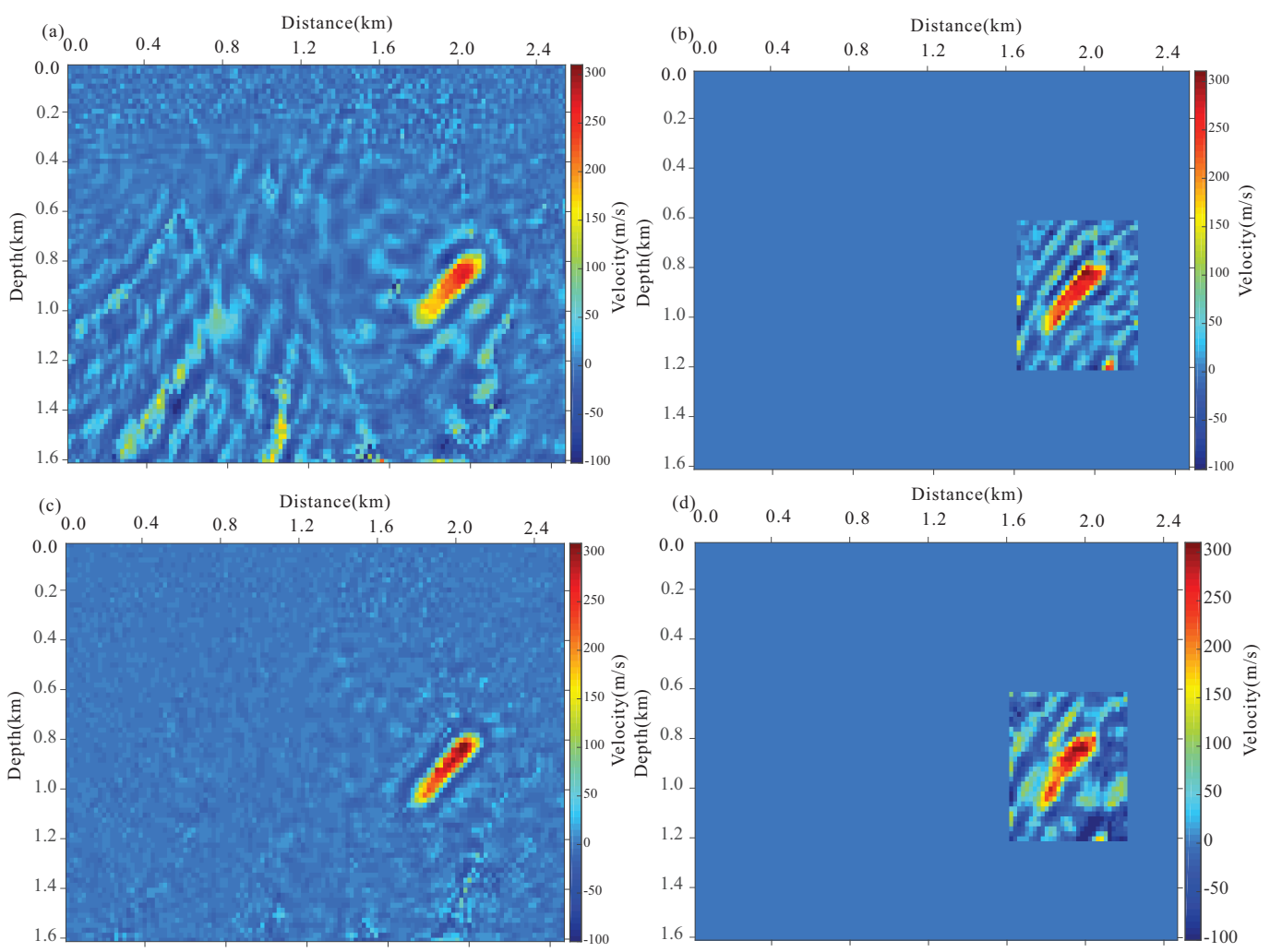

Figure 9: The time-lapse inversion results of noisy data $(30 \mathrm{~dB})$ for a perturbation of $300 \mathrm{~m} / \mathrm{s}$ with the inverted baseline model using the same noise-level data as the starting model. (a) The full-model inversion with the sequential difference strategy; (b) the target-oriented inversion with the sequential difference strategy; (c) the full-model inversion with the double difference strategy; (d) the target-oriented inversion with the double difference strategy.

$\%$ to $80 \%$. The reason why the computation time is different is that because of the use of different strategies, the computation stops at different iterations for different frequencies. The results in Fig. 10 show clear difference for four numerical experiments from different inversion scheme. For the sequential difference strategy, the inverted time-lapse velocity perturbation from the target-oriented scheme appears more clear, however, the result from the full-model scheme with strong noise is not so clear. The situation improves significantly for the double difference strategy shown in Figs. 10(c) and (d). Also, for the double difference strategy, the results with noisy data from the full-model inversion are slightly better than that from the target-oriented inversion.

Furthermore, we have tested a smaller time-lapse perturbation of $100 \mathrm{~m} / \mathrm{s}$. Figs. 11(a) and $(b)$ show the inverted results of noisy data $(26 \mathrm{~dB})$ with the double difference and sequential difference strategies. From Figs. 11(a) and (b), one can observe the following features of the inverted time-lapse perturbation: (1) although the time-lapse perturbation can be displayed clearly, there are some artifacts outside the region of time-lapse velocity perturbation; (2) compared with the inverted results of the perturbation of $300 \mathrm{~m} / \mathrm{s}$, the 

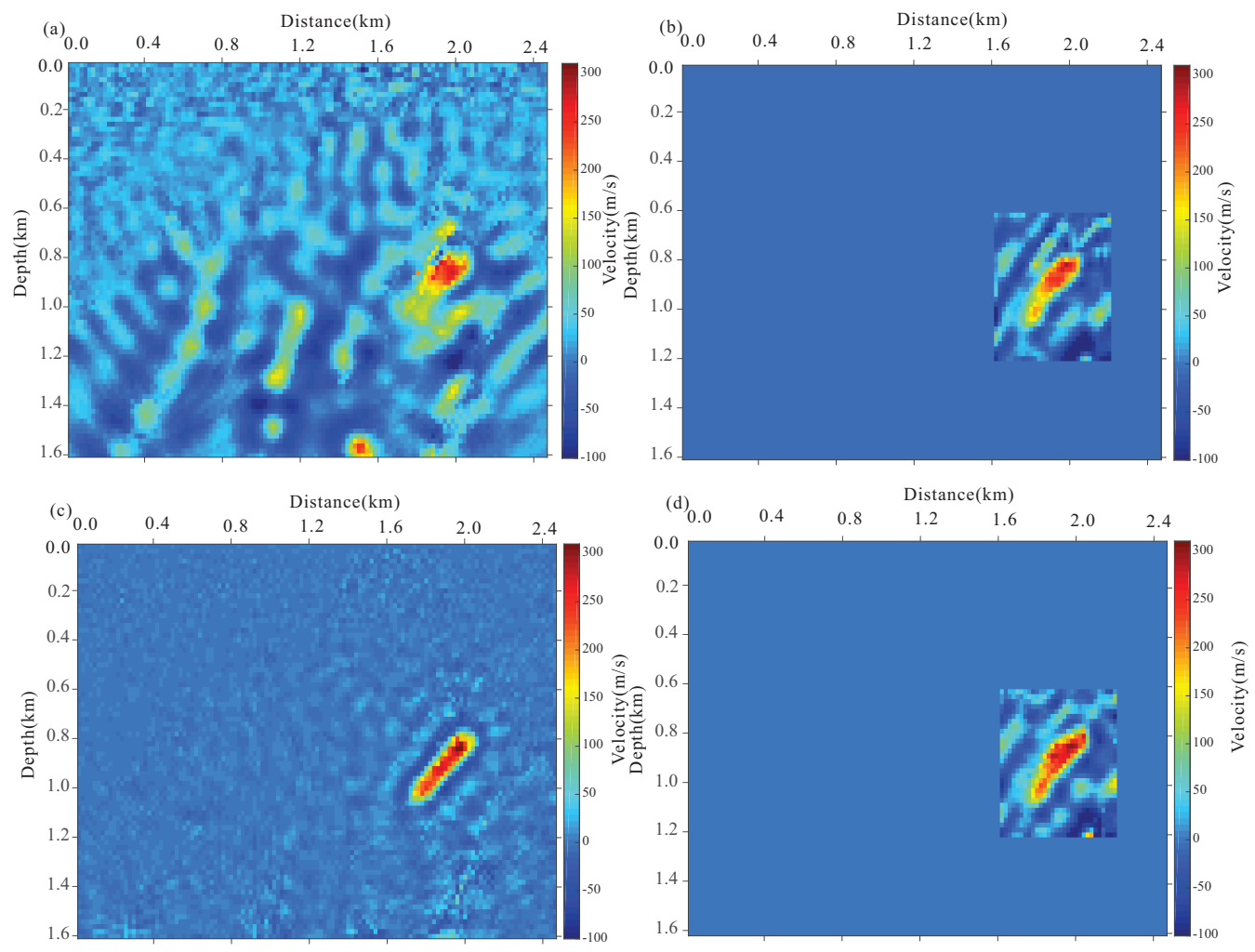

Figure 10: The time-lapse inversion results of noisy data $(26 \mathrm{~dB})$ for a perturbation of $300 \mathrm{~m} / \mathrm{s}$ with the inverted baseline model using the same noise-level data as the starting model. (a) The full-model inversion with the sequential difference strategy; (b) the target-oriented inversion with the sequential difference strategy; (c) the full-model inversion with the double difference strategy; (d) the target-oriented inversion with the double difference strategy.
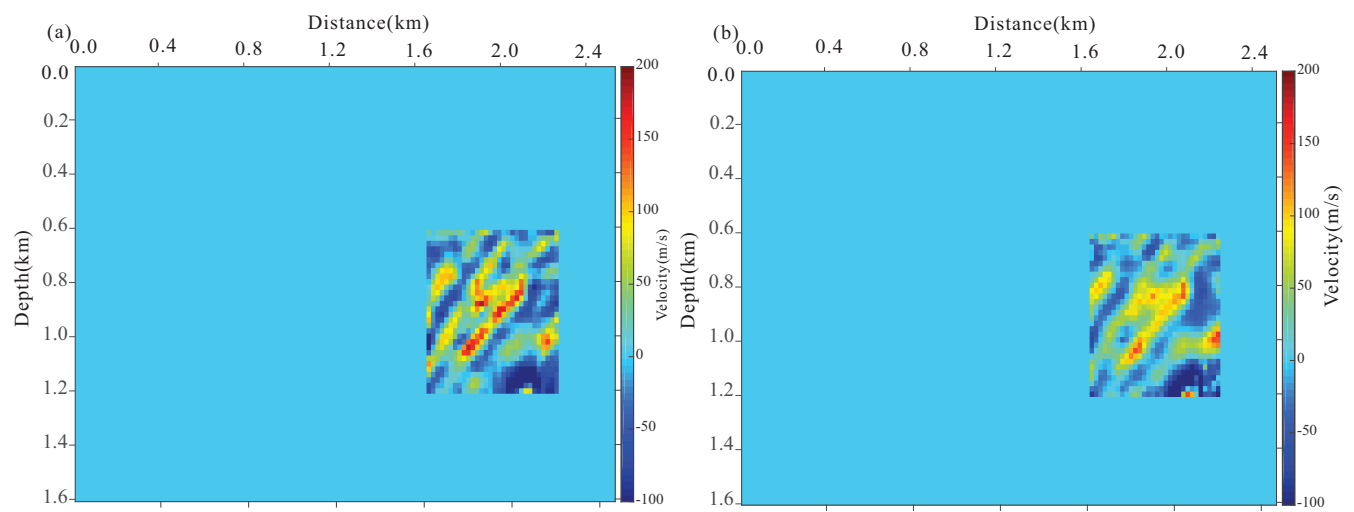

Figure 11: The time-lapse inversion results of noisy data $(26 \mathrm{~dB})$ for a perturbation of $100 \mathrm{~m} / \mathrm{s}$ with the inverted baseline model using the same noise-level data. (a) The target-oriented inversion with the sequential difference strategy; (b) the target-oriented inversion with the double difference strategy. 
inverted results of the perturbation of $100 \mathrm{~m} / \mathrm{s}$ look less accurate. This indicates that the smaller value of the time-lapse velocity perturbation we have, the more difficult for inversion.

\section{Conclusions}

Full waveform inversion can be an effective tool for time-lapse seismic data because of its applicability of extracting information on subsurface structure. However, the inversion of the time-lapse seismic waveform data requires to repeat computations for monitor survey. This renders the inversion very expensive for the reservoir monitor. Instead of performing a full-model inversion, we focus on inversion of a target region, where the reservoir changes occur. Jakobsen and Ursin [24] mentioned the possibility of doing T-matrix based time-lapse inversion, but never did this. The main finding is that we have verified that the T-matrix approach is indeed naturally target-oriented and allows one to reduce the computational cost of time-lapse inversion by focusing the inversion on the target-area only. Our approach is based on the DBIT method using the integral equation form. In our scheme, the Gaussian beam has been introduced, which allows to compute the wavefields from the surface to the target region in the smoothed baseline model. Then, by combining the background Green's function using the Gaussian beam with the T-matrix, we obtain the Green's function for the time-lapse inversion. For the local inversion of the target region, the T-matrix is used to update the wavefields within the target region.

Numerical examples are presented, showing that because of the limited size of the target-oriented time-lapse inversion, the computational cost has been reduced by approximately $80 \%$. However, for the double difference strategy, the results with noise-free data from the target-oriented inversion are better than that from the full-model inversion, while the results with noisy data from the full-model inversion are slightly better than that from the target-oriented inversion. That means that the double difference strategy is relatively sensitive to the random noise. For the sequential difference strategy, compared with the results from the full-model inversion, the results of the time-lapse model from the target-oriented inversion are nearly the same. This method should be possible, due to the computational efficiency, to perform a 4-D seismic full waveform inversion. Future works will be also directed toward to a 4-D inversion of the time-lapse waveform data and extended by the domain decomposition method [31].

\section{Acknowledgments}

The authors acknowledge the Research Council of Norway for the Petromaks II project 267769 (Bayesian inversion of 4D seismic waveform data for quantitative integration with production data). The third author acknowledges the Research Council of Norway and the industry partners; ConocoPhillips Skandinavia AS, Aker BP ASA, Eni Norge AS, 
Maersk Oil Norway AS, Statoil Petroleum AS, Neptune Energy Norge AS, Lundin Norway AS, Halliburton AS, Schlumberger Norge AS, Wintershall Norge AS and DEA Norge AS of The National IOR Centre of Norway for support.

\section{Appendix A: The Green's function using Gaussian beam summation}

The Green's function can be computed by a Gaussian beam summation [40,78]

$$
G^{(0)}\left(\mathbf{x}, \mathbf{x}^{\prime}, \omega\right)=\frac{i}{4 \pi} \int \frac{d p_{x}}{p_{z}} \mathbf{u}_{G B}\left(\mathbf{x}^{\prime} ; \mathbf{x}, \mathbf{p} ; \omega\right),
$$

where, $\mathbf{x}$ is the source (starting point) position vector, $\mathbf{x}^{\prime}$ is the subsurface scattering point vector, the ray parameter vector $\mathbf{p}$ is defined by

$$
\mathbf{p}=\left\{p_{x}, p_{z}\right\},
$$

where $p_{x} p_{z}$ are ray parameters, respectively. Further, $u_{G B}$ is a single Gaussian beam $[40,41]$

$$
\begin{aligned}
\mathbf{u}_{G B}\left(q_{1}, q_{2}, \tau\right) & =U^{\Omega}(\tau)(\operatorname{det} W)^{1 / 2} \exp \left[-\frac{1}{2} \omega q^{T} \operatorname{Im} M(\tau) q\right] \\
& =\exp \left\{-i \omega\left[t-T(\tau)-\frac{1}{2} q^{T} \operatorname{Im} M(\tau) q\right]\right\}
\end{aligned}
$$

with

$$
W\left(\tau_{0}, \tau\right)=Q_{1}\left(\tau_{0}, \tau\right)+Q_{2}\left(\tau_{0}, \tau\right)\left[\operatorname{Re} M\left(\tau_{0}\right)+i \operatorname{Im} M\left(\tau_{0}\right)\right],
$$

where $T(\tau)$ the traveltime along ray, $Q_{1}\left(\tau_{0}, \tau\right)$ and $Q_{2}\left(\tau_{0}, \tau\right)$ are real-valued matrices, which are solutions of the dynamic ray tracing system in matrix form, $q=\left(q_{1}, q_{2}\right)^{T}$ and $q_{1}, q_{2}$ are the ray-centred coordinates, which can be constructed along the ray $\Omega$. Moreover $M\left(\tau_{0}\right)$ is the $2 \times 2$ complex-valued matrix, which represents the second-order derivatives of the ray traveltime. Following Hill $[79,80]$, the sampling for the horizontal slowness $d p_{x}$ can be chosen as

$$
d p_{x}=\frac{1}{6 l \sqrt{f_{\min }, f_{\max }}}
$$

where $f_{\min }$ and $f_{\max }$ are the lowest and highest frequencies, respectively. The initial beam width can be chosen as

$$
l=\frac{V_{a v g}}{f_{\min }}
$$

where $V_{\text {avg }}$ is the average velocity of the full model. 


\section{Appendix B}

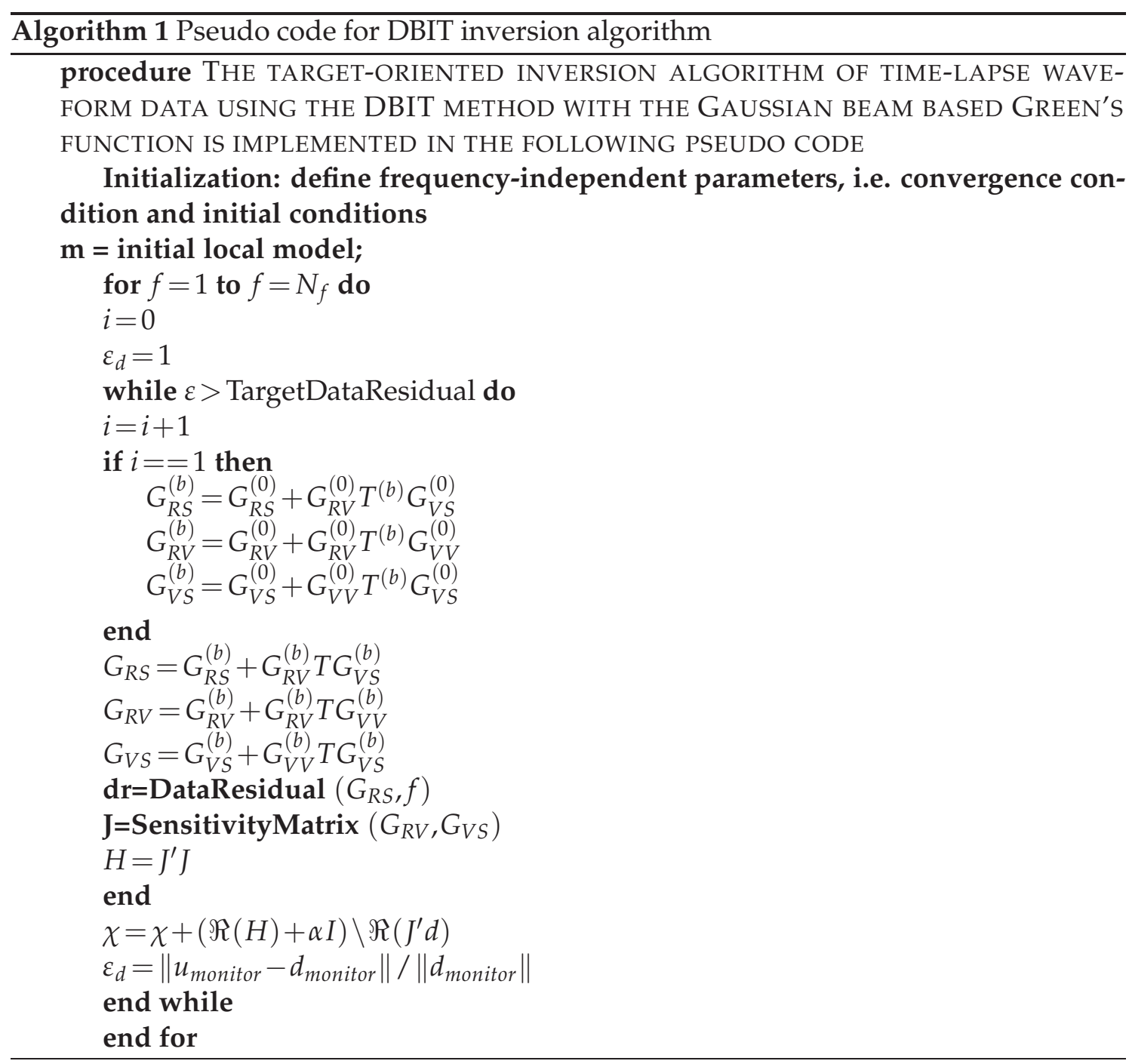

\section{References}

[1] Virieux, J. and Operto, S. An overview of full-waveform inversion in exploration geophysics, Geophysics, 74 (2009), WCC1-WCC26.

[2] Landrø, M., and Stammeijer, J. Quantitative estimation of compaction and velocity changes using 4D impedance and traveltime changes. Geophysics, 69 (2004), 949-957.

[3] Lumley, D. 4D seismic monitoring of CO2 sequestration. The Leading Edge, 29 (2010), 150155. 
[4] Maharramov, M., Biondi, B. L., and Meadows, M. A. Time-lapse inverse theory with applications. Geophysics, 81 (2016), R485-R501.

[5] Zheng, Y., Barton, P., and Singh, S. Strategies for elastic full-waveform inversion of timelapse ocean bottom cable (OBC) seismic data: 81st Annual International Meeting, SEG, Expanded Abstracts (2011), 4195-4200.

[6] Routh, P., Palacharla, G., Chikichev, I., and Lazaratos, S. Full wavefield inversion of timelapse data for improved imaging and reservoir characterization: 82nd Annual International Meeting, SEG, Expanded Abstracts, (2012), 1-6.

[7] Asnaashari, A., Brossier, R., Garambois, S., Audebert, F., Thore, P., and Virieux, J. Timelapse seismic imaging using regularized full waveform inversion with a prior model: which strategy? Geophys. Prospect. 63 (2015), 78-98.

[8] Yang, D., Liu, F., Morton, S., et al. Time-lapse full-waveform inversion with ocean-bottomcable data: Application on Valhall field. Geophysics 81 (2016), R225-R235.

[9] Kamei, R., and Lumley, D. Full waveform inversion of repeating seismic events to estimate time-lapse velocity changes. Geophysical Journal International, 209 (2017), 1239-1264.

[10] Yuan, S., Fuji, N., Singh, S., and Borisov, D. Localized time-lapse elastic waveform inversion using wavefield injection and extrapolation: 2-D parametric studies. Geophysical Journal International, 209 (2017), 1699-1717.

[11] Tarantola, A., Inversion of seismic reflection data in the acoustic approximation: Geophysics, 34 (1984), 1259-1266.

[12] Tarantola, A., A strategy for nonlinear elastic inversion of seismic reflection data: Geophysics, 51 (1986), 1893-1903.

[13] Oristaglio M. L. and Blok H. Wavefield Imaging and Inversion in Electromagnetics and Acoustics. Cambridge University Press, 2012.

[14] Pike R. and Sabatier P. Scattering and inverse scattering in pure and applied science. Academic Press, 2002.

[15] Weglein, A. B., Araujo, F. V., Carvalho, P. M., Stolt, R. H., Matson, K. H., Coates, R. T., and Zhang, H. Inverse scattering series and seismic exploration. Inverse problems, 19 (2003), R27.

[16] Qu, Y., Li, Z., Huang, J., Li, J., and Guan, Z. Elastic full-waveform inversion for surface topography. Geophysics, 82 (2017), R269-R285.

[17] Hu, Y., Han, L. G., Wu, R. S., Feng, Q. Multi-scale time-frequency domain full waveform inversion with a local correlation-phase misfit function. In SEG Technical Program Expanded Abstracts 2018 (pp. 1058-1062). Society of Exploration Geophysicists.

[18] Clayton, R. W., and Stolt, R. H. A Born-WKBJ inversion method for acoustic reflection data. Geophysics, 46 (1981), 1559-1567.

[19] Cohen, J. K., and Bleistein, N. Velocity inversion procedure for acoustic waves. Geophysics, 44 (1979), 1077-1087.

[20] Weglein, A. B., Boyse, W. E., and Anderson, J. E. Obtaining three-dimensional velocity information directly from reflection seismic data: An inverse scattering formalism. Geophysics, 46 (1981), 1116-1120.

[21] Berkhout, A. J. Seismic resolution: A quantitative analysis of resolving power of acoustical echo techniques (Vol. 12). Geophysical Press, (1984).

[22] Beylkin, G. Imaging of discontinuities in the inverse scattering problem by inversion of a causal generalized Radon transform. Journal of Mathematical Physics, 26 (1985), 99-108.

[23] Stolt, R. H., and Weglein, A. B. Migration and inversion of seismic data. Geophysics, 50 (1985), 2458-2472. 
[24] Jakobsen, M. and Ursin, B. Full waveform inversion in the frequency domain using direct iterative T-matrix methods, J. Geophys. Eng., 12 (2015), 400-418.

[25] Tao, Y. and Sen, M. K. Frequency-domain full waveform inversion with a scatteringintegral approach and its sensitivity analysis. Journal of Geophysics and Engineering, 10 (2013), 065008.

[26] Wang, Y. M., and Chew, W. C. Accelerating the iterative inverse scattering algorithms by using the fast recursive aggregate T-matrix algorithm. Radio science, 27 (1992), 109-116.

[27] Kouri, D. J., and Vijay, A. Inverse scattering theory: Renormalization of the LippmannSchwinger equation for acoustic scattering in one dimension. Physical Review E, 67 (2003), 046614 .

[28] $\mathrm{Wu}, \mathrm{R}$. S. and Zheng, Y. Nonlinear partial derivative and its DeWolf approximation for nonlinear seismic inversion Geophys. J. Int. 196 (2014), 1827-43.

[29] Jakobsen, M., and Wu, R. S. Renormalized scattering series for frequency-domain waveform modelling of strong velocity contrasts: Geophysical Journal International, 206 (2016), 880-899.

[30] Jakobsen, M., Wu, R. S., Huang, X. Seismic waveform modeling in strongly scattering media using renormalization group theory. In SEG Technical Program Expanded Abstracts 2018 (pp. 5007-5011). Society of Exploration Geophysicists.

[31] Jakobsen, M. and Wu, R. S. Accelerating the T-matrix approach to seismic full-waveform inversion by domain decomposition. Geophysical Prospecting, 66 (2018), 1039-1059.

[32] Wang, B., Jakobsen, M., Wu, R.S., Lu, W. and Chen, X. Accurate and efficient velocity estimation using Transmission matrix formalism based on the decomposition method. Inverse problems, 33 (2017), 035002 (13pp).

[33] Eikrem, K. S., Jakobsen, M. and Nævdal, G. Bayesian Inversion of Time-lapse Seismic Waveform Data Using an Integral Equation Method. In: IOR 2017-19th European Symposium on Improved Oil Recovery, (2017).

[34] Kirchner, A. and Shapiro, S. A. Fast repeat-modelling of time-lapse seismograms. Geophysical prospecting, 49 (2001), 557-569.

[35] Borisov, D., Singh, S. C., and Fuji, N. An efficient method of 3-D elastic full waveform inversion using a finite-difference injection method for time-lapse imaging. Geophysical Journal International, 202 (2015), 1908-1922.

[36] Willemsen, B., Malcolm, A., and Lewis, W. A numerically exact local solver applied to salt boundary inversion in seismic full-waveform inversion. Geophysical Journal International, 204 (2016), 1703-1720.

[37] Malcolm, A., and Willemsen, B. Rapid 4D FWI using a local wave solver. The Leading Edge, 35 (2016), 1053-1059.

[38] Broggini, F., Vasmel, M., Robertsson, J. O., and van Manen, D. J. Immersive boundary conditions: Theory, implementation, and examples. Geophysics, 82 (2017), T97-T110.

[39] Willemsen, B., and Malcolm, A., An efficient coupled acoustic-elastic local solver applied to phase inversion. Geophysics, 82 (2017), R219-R234.

[40] Cerveny, V. Seismic ray theory. Cambridge university press, (2001).

[41] Cerveny, V., Popov M. M., and Psencik, I. Computation of wave fields in inhomogeneous media-Gaussian beam approach. Geophysical Journal International, 70 (1982), 109-128.

[42] Huang, X. and Greenhalgh, S. Linearized formulations and approximate solutions for the complex eikonal equation in orthorhombic media and applications of complex seismic traveltime. Geophysics 83 (2018), C115-C136.

[43] Huang, X. and Sun, H. Numerical modelling of Gaussian beam propagation and diffrac- 
tion in inhomogeneous media based on the complex eikonal equation. Acta Geophysica, 4 (2018), 497-508.

[44] Huang, X., Sun, J. and Greenhalgh, S. On the solution of the complex eikonal equation in acoustic VTI media: A perturbation plus optimization scheme. Geophysical Journal International, 214 (2018), 907-932.

[45] Huang, X, Sun, H., and Sun, J. Born modeling for heterogeneous media using the Gaussian beam summation based Green's function. Journal of Applied Geophysics, 131 (2016), 191201.

[46] Huang, X., Sun, J., and Sun, Z. Local algorithm for computing complex travel time based on the complex eikonal equation. Physical Review E, 93 (2016), 043307.

[47] Huang, X. Extended Beam Approximation for High-Frequency Wave Propagation. IEEE Access, 6 (2018), 37214-37224.

[48] Chew et al. Integral Equation Methods for Electromagnetic and Elastic Waves. San Rafael, CA, USA: Morgan and Claypool, (2008).

[49] Levinson, H.W. and Markel, V. A. Solution to the nonlinear inverse scattering problem by T-matrix completion. I. Theory. Physical Review E 94 (2016a), 043317.

[50] Levinson, H.W. and Markel, V. A. Solution to the nonlinear inverse scattering problem by T-matrix completion. II.Simulations. Physical Review E 94 (2016b), 043318.

[51] March, N.H., Young, W.H. and Sampanthar, S. The Many-Body Problem in Quantum Mechanics. Dover Publications Inc., (1967) New York.

[52] Markel, V. A., and Schotland, J. C. On the convergence of the Born series in optical tomography with diffuse light. Inverse problems 23 (2007), 1445.

[53] Newton, R.G. Scattering Theory of Waves and Particles. Dover Publications Inc., (2002), New York.

[54] Tsang, L., and Kong, J. A.. Multiple scattering of electromagnetic waves by random distributions of discrete scatterers with coherent potential and quantum mechanical formalism. Journal of Applied Physics 51 (1980), 3465-3485.

[55] Gonis, A. and Butler, W. H. Multiple Scattering in Solids. Springer-Verlag, (2000), New York.

[56] Jakobsen, M. T-matrix approach to seismic forward modelling in the acoustic approximation. Studia Geophysica et Geodaetica, 56 (2012), 1-20.

[57] Jakobsen, M., and Hudson J. A. Visco-elastic waves in rock-like composites. Studia Geophysica et Geodaetica 47 (2003), 793-826.

[58] Jakobsen, M., Hudson, J. A., and Johansen T. A. T-matrix approach to shale acoustics. Geophysical Journal International 154 (2003), 533-558.

[59] Morse, P.M. and Feshbach, H. Methods of theoretical physics, (1953), McGraw-Hill New York.

[60] Chew, W. C., and Wang, Y. M. Reconstruction of two-dimensional permittivity distribution using the distorted Born iterative method. IEEE transactions on medical imaging, 9 (1990), 218-225.

[61] Wang, Y. M., and Chew, W. C. Accelerating the iterative inverse scattering algorithms by using the fast recursive aggregate Tmatrix algorithm. Radio science, 27 (1992), 109-116.

[62] Tikhonov, Andrei Nikolaevitch, et al. Numerical methods for the solution of ill-posed problems. Vol. 328. (2013) Springer Science Business Media.

[63] Lavarello R. J. and. Oelze M. L, Density imaging using inverse scattering, J. Acoust. Soc. Am., vol. 125, no. 2, (2009) pp. 793-802.

[64] Hesford, A. J., and Chew, W. C. Fast inverse scattering solutions using the distorted Born 
iterative method and the multilevel fast multipole algorithm. The Journal of the Acoustical Society of America, 128 (2010), 679-690.

[65] Hansen, P. C., and O'Leary, D. P. The use of the L-curve in the regularization of discrete ill-posed problems. SIAM Journal on Scientific Computing, 14 (1993), 1487-1503.

[66] Waldhauser, F., and Ellsworth, W. L. A double-difference earthquake location algorithm: Method and application to the northern Hayward fault, California. Bulletin of the Seismological Society of America, 90 (2000), 1353-1368.

[67] Denli, H., and Huang, L. Double-difference elastic waveform tomography in the time domain. SEG Annual Meeting. Society of Exploration Geophysicists, (2009).

[68] Zhang, Z., and Huang, L. Double-difference elastic-waveform inversion with prior information for time-lapse monitoring. Geophysics, 78 (2013), R259-R273.

[69] Tang, Y., Target-oriented wave-equation least-squares migration/inversion with phaseencoded Hessian. Geophysics, 74 (2009), WCA95-WCA107.

[70] Valenciano, A. A., Biondi, B., and Guitton, A. Target-oriented wave-equation inversion. Geophysics, 71 (2006), A35-A38.

[71] Luo, Y., and Schuster, G. T. Bottom-up target-oriented reverse-time datuming: CPS. In SEG Geophysics Conference and Exhibition F (Vol. 55), (2004).

[72] Martin, G.S., Wiley, R. and Marfurt, K.J. Marmousi2: An elastic upgrade for Marmousi. The Leading Edge, 25 (2006), 156-166.

[73] Oristaglio, M. L. and Blok, H. Wavefield Imaging and Inversion in Electromagnetics and Acoustics, Cambridge, Cambridge University Press, (2012).

[74] Remis, R. F. and van den Berg, P. M. On the equivalence of the Newton-Kantorovich and distorted Born iterative methods Inverse Problems, 16 (2000), L1-4.

[75] Métivier, L., Brossier, R., Virieux, J., and Operto, S. Full waveform inversion and the truncated Newton method. SIAM Journal on Scientific Computing 35 (2013): B401-B437.

[76] Pan, W., Kristopher A. Innanen, and W. Liao. Accelerating Hessian-free Gauss-Newton full-waveform inversion via l-BFGS preconditioned conjugate-gradient algorithm. Geophysics 82 (2017), R49-R64.

[77] Yang, D., Zheng, Y., Fehler, M., and Malcolm, A. Target-oriented time-lapse waveform inversion using virtual survey, in 2012 SEG Annual Meeting, Society of Exploration Geophysicists, (2012).

[78] Gray, S. H., Bleistein, N. True-amplitude Gaussian-beam migration. Geophysics, 74 (2009), S11-S23.

[79] Hill, N. R. Gaussian beam migration. Geophysics, 55 (1990), 1416-1428.

[80] Hill, N. R. Prestack Gaussian-beam depth migration. Geophysics, 66 (2001), 1240-1250.

[81] Jakobsen, M., and Tveit, S. Distorted Born iterative T-matrix method for inversion of CSEM data in anisotropic media. Geophysical Journal International. 214 (2018), 1524-1537. 